PROCEEDINGS OF THE

AMERICAN MATHEMATICAL SOCIETY

Volume 128, Number 2, Pages 353-360

S 0002-9939(99)05029-7

Article electronically published on July 6, 1999

\title{
A NOTE ON $p$-BASES OF RINGS
}

\author{
TOMOAKI ONO
}

(Communicated by Wolmer V. Vasconcelos)

\begin{abstract}
Let $R \supseteq R^{\prime} \supseteq R^{p}$ be a tower of rings of characteristic $p>0$. Suppose that $R$ is a finitely presented $R^{\prime}$-module. We give necessary and sufficient conditions for the existence of $p$-bases of $R$ over $R^{\prime}$. Next, let $A$ be a polynomial ring $k\left[X_{1}, \ldots, X_{n}\right]$ where $k$ is a perfect field of characteristic $p>0$, and let $B$ be a regular noetherian subring of $A$ containing $A^{p}$ such that $\left[Q(B): Q\left(A^{p}\right)\right]=p$. Suppose that $\operatorname{Der}_{A^{p}}(B)$ is a free $B$-module. Then, applying the above result to a tower $B \supseteq A^{p} \supseteq B^{p}$ of rings, we shall show that a polynomial of minimal degree in $B-A^{p}$ is a $p$-basis of $B$ over $A^{p}$.
\end{abstract}

\section{Preliminaries}

Throughout this paper, let $p$ be always a prime number, let $R$ be a commutative ring with unity of characteristic $p$, and let $R^{\prime}$ be a subring of $R$ containing $R^{p}=$ $\left\{a^{p} \mid a \in R\right\}$. Then there is a canonical one-to-one correspondence between $\operatorname{Spec} R$ and Spec $R^{\prime}$ by Lemma 1 of [6]. So, for any given $\mathfrak{p} \in \operatorname{Spec} R$, we denote by $\mathfrak{p}^{\prime}$ the corresponding element in $\operatorname{Spec} R^{\prime}$, i.e., $\mathfrak{p}^{\prime}=\mathfrak{p} \cap R^{\prime}$.

A subset $\left\{x_{1}, \ldots, x_{l}\right\}$ of $R$ is said to be a $p$-basis of $R$ over $R^{\prime}$ if the monomials $x_{1}^{e_{1}} \cdots x_{l}^{e_{l}}\left(0 \leq e_{i} \leq p-1\right)$ are linearly independent over $R^{\prime}$ and $R=R^{\prime}\left[x_{1}, \ldots, x_{l}\right]$. If, for each $\mathfrak{p} \in \operatorname{Spec} R$, there exists a $p$-basis of $R_{\mathfrak{p}}$ over $R_{\mathfrak{p}^{\prime}}^{\prime}$, we say that $R$ has locally $p$-bases over $R^{\prime}$. Moreover, if $R$ is finitely generated and projective as an $R^{\prime}$-module in addition to the previous condition, then the $R^{\prime}$-algebra $R$ is called $a$ Galois extension of $R^{\prime}([6])$.

When $R$ is a local ring, the existence of a $p$-basis of $R$ over $R^{\prime}$ is studied for example in [1]. But it is not well-known whether there is a $p$-basis of $R$ over $R^{\prime}$ or not, when $R$ is not a local ring ([2]). If $R$ has a $p$-basis over $R^{\prime}$, then for any $\mathfrak{p} \in S p e c R$ the localization $R_{\mathfrak{p}}$ at $\mathfrak{p}$ also has a $p$-basis over $R_{\mathfrak{p}^{\prime}}^{\prime}$. The converse does not hold in general. In this paper, we study a condition for the existence of a $p$-basis of $R$ over $R^{\prime}$, when $R$ has locally p-bases over $R^{\prime}$ (Theorems 2.2 and 3.2). As an example we consider the existence of a $p$-basis of a regular ring which is contained in a polynomial ring over a perfect field (Theorem 4.1). A special basis of the module of derivations plays a central role in our study, and we use the results of [6] frequently.

Let $\operatorname{Der}_{R^{\prime}}(R)$ be the set of all derivations of $R$ over $R^{\prime}$, let $S$ be a multiplicatively closed subset of $R$, and let $S^{\prime}$ be $S \cap R^{\prime}$. We denote by $\phi_{S}$ and $\tau_{S}$ the canonical

Received by the editors January 19, 1998 and, in revised form, April 1, 1998.

1991 Mathematics Subject Classification. Primary 13N05; Secondary 13B05.

Key words and phrases. p-basis, derivation, Galois extension.

(C)1999 American Mathematical Society 
maps $R \longrightarrow R_{S}$ and $\operatorname{Der}_{R^{\prime}}(R) \longrightarrow \operatorname{Der}_{R_{S^{\prime}}^{\prime}}\left(R_{S}\right)$, respectively. In particular, when $S$ is a multiplicatively closed subset $\left\{f^{n}\right\}_{n \geq 0} \quad(f \in R)$, resp. $R-\mathfrak{p}$, we denote by $\phi_{f}$ and $\tau_{f}$, resp. $\phi_{\mathfrak{p}}$ and $\tau_{\mathfrak{p}}$ (or simply $\phi$ and $\tau$ ), the previous canonical maps. Note that $\tau_{S}(D)\left(\phi_{S}(x)\right)=\phi_{S}(D(x))$ for any $x \in R$ and any $D \in \operatorname{Der}_{R^{\prime}}(R)$.

As is well-known, the following three facts hold:

(1) If $R_{S}$ has a $p$-basis $\left\{x_{1} / s_{1}, \ldots, x_{l} / s_{l}\right\}$ over $R_{S^{\prime}}^{\prime}$, then $\left\{\phi_{S}\left(s_{1}^{p-1} x_{1}\right), \ldots\right.$, $\left.\phi_{S}\left(s_{l}^{p-1} x_{l}\right)\right\}$ is a $p$-basis of $R_{S}$ over $R_{S^{\prime}}^{\prime}$, i.e., we can choose a $p$-basis of $R_{S}$ over $R_{S^{\prime}}^{\prime}$ from the image $\phi(R)$.

(2) If $R$ has a $p$-basis $\left\{x_{1}, \ldots, x_{l}\right\}$ over $R^{\prime}$, then the image $\left\{\phi_{S}\left(x_{1}\right), \ldots, \phi_{S}\left(x_{l}\right)\right\}$ in $R_{S}$ is a $p$-basis of $R_{S}$ over $R_{S^{\prime}}^{\prime}$.

(3) If $R$ has a $p$-basis $\left\{x_{1}, \ldots, x_{l}\right\}$ over $R^{\prime}$, then there exists a unique set of derivations $D_{x_{1}}, \ldots, D_{x_{l}}$ of $R$ over $R^{\prime}$ such that $D_{x_{i}}\left(x_{j}\right)=\delta_{i j}$ where $\delta_{i j}$ is Kronecker's delta. This set forms a basis for $\operatorname{Der}_{R^{\prime}}(R)$. We always denote by $D_{x_{1}}, \ldots, D_{x_{l}}$ such derivations which are associated with a $p$-basis $\left\{x_{1}, \ldots, x_{l}\right\}$ of $R$ over $R^{\prime}$.

Definition. Suppose $R$ has locally $p$-bases over $R^{\prime}$. Let $\{\mathfrak{m}\}$ be the set of all maximal ideals of $R$. We call $D \in D r_{R^{\prime}}(R)$ the preferable derivation, if for each $\mathfrak{m}$ there is a $p$-basis $\left\{\phi_{\mathfrak{m}}(x)\right\}(x \in R)$ of $R_{\mathfrak{m}}$ over $R_{\mathfrak{m}^{\prime}}^{\prime}$ such that $\phi_{\mathfrak{m}}(D(x))^{p-1} \in$ $\bigoplus_{i=0}^{p-2} R_{\mathfrak{m}^{\prime}}^{\prime} \phi_{\mathfrak{m}}(x)^{i}$.

Lemma 1.1. Suppose $R$ has locally p-bases over $R^{\prime}$. Let $S$ be a multiplicatively closed subset of $R$ disjoint from at least one prime ideal, and suppose $R_{S}$ has a $p$-basis $\left\{\phi_{S}(x)\right\}$ over $R_{S^{\prime}}^{\prime}$. If $D \in D e r_{R^{\prime}}(R)$ is preferable, then $\phi_{S}(D(x))^{p-1} \in$ $\bigoplus_{i=0}^{p-2} R_{S^{\prime}}^{\prime} \phi_{S}(x)^{i}$.

Proof. Let $\{\mathfrak{p}\}$ be the set of all prime ideals of $R$ disjoint from $S$. The set $\{\mathfrak{p}\}$ is non-empty by the assumption. Let $\mathfrak{m}$ be a maximal ideal containing $\mathfrak{p}$. Since $D$ is preferable, there exists a $p$-basis $\left\{\phi_{\mathfrak{m}}(y)\right\}(y \in R)$ of $R_{\mathfrak{m}}$ over $R_{\mathfrak{m}^{\prime}}^{\prime}$ such that $\phi_{\mathfrak{m}}(D(y))^{p-1} \in \bigoplus_{i=0}^{p-2} R_{\mathfrak{m}^{\prime}}^{\prime} \phi_{\mathfrak{m}}(y)^{i}$. We use a symbol $\phi$ for the canonical map $R \longrightarrow R_{\mathfrak{p}}$. Then, by the above fact $(2),\{\phi(y)\}$ is a $p$-basis of $R_{\mathfrak{p}}$ over $R_{\mathfrak{p}^{\prime}}^{\prime}$, and $\phi(D(y))^{p-1} \in \bigoplus_{i=0}^{p-2} R_{\mathfrak{p}^{\prime}}^{\prime} \phi(y)^{i}$. Therefore we can take an element $z$ of $R_{\mathfrak{p}}$ such that $\phi(D(y))^{p-1}=D_{\phi(y)}(z)$. Since $\left\{D_{\phi(y)}\right\}$ forms a basis for $\operatorname{Der}_{R_{\mathfrak{p}^{\prime}}}\left(R_{\mathfrak{p}}\right)$ and $D_{\phi(y)}(\phi(y))=1$, we have $\tau_{\mathfrak{p}}(D)=\tau_{\mathfrak{p}}(D)(\phi(y)) D_{\phi(y)}=\phi(D(y)) D_{\phi(y)}$.

Now, the fact (2) says that $\{\phi(x)\}$ is a $p$-basis of $R_{\mathfrak{p}}$ over $R_{\mathfrak{p}^{\prime}}^{\prime}$. Hence, there is a unique basis $\left\{D_{\phi(x)}\right\}$ of $\operatorname{Der}_{R_{\mathfrak{p}^{\prime}}^{\prime}}\left(R_{\mathfrak{p}}\right)$ such that $D_{\phi(x)}(\phi(x))=1$, and $D_{\phi(y)}=$ $D_{\phi(y)}(\phi(x)) D_{\phi(x)}$. From these facts, we get the following equations:

$$
\begin{aligned}
\phi(D(x))^{p-1} & =\left\{\tau_{\mathfrak{p}}(D)(\phi(x))\right\}^{p-1} \\
& =\left\{\phi(D(y)) D_{\phi(y)}(\phi(x))\right\}^{p-1} \\
& =D_{\phi(y)}(z) D_{\phi(y)}(\phi(x))^{p-1} \\
& =\left\{D_{\phi(y)}(\phi(x)) D_{\phi(x)}(z)\right\} D_{\phi(y)}(\phi(x))^{p-1} \\
& =D_{\phi(y)}(\phi(x))^{p} D_{\phi(x)}(z) .
\end{aligned}
$$

Thus $\phi(D(x))^{p-1}$ is contained in $\bigoplus_{i=0}^{p-2} R_{\mathfrak{p}^{\prime}}^{\prime} \phi(x)^{i}$. When we write $\phi_{S}(D(x))^{p-1}$ as $\sum_{i=0}^{p-1}\left(c_{i} / s_{i}\right) \phi_{S}(x)^{i}\left(c_{i} \in R^{\prime}, s_{i} \in S^{\prime}\right)$, we can find for each $\mathfrak{p}$ an element $t$ of $R^{\prime}-\mathfrak{p}^{\prime}$ such that $c_{p-1} t=0$, i.e., $\phi\left(c_{p-1}\right)=0$. This implies that $\phi_{S}\left(c_{p-1}\right)=0$. Therefore $\phi_{S}(D(x))^{p-1} \in \bigoplus_{i=0}^{p-2} R_{S^{\prime}}^{\prime} \phi_{S}(x)^{i}$. 
Lemma 1.2. Suppose $R$ is reduced and has locally p-bases over $R^{\prime}$. Let $\{\mathfrak{q}\}$ be the set of all minimal prime ideals belonging to the zero ideal $(0)$, and let $\left\{\phi_{\mathfrak{q}}(x)\right\}$ $(x \in R)$ be a p-basis of $R_{\mathfrak{q}}$ over $R_{\mathfrak{q}^{\prime}}$. If $D \in D r_{R^{\prime}}(R)$ satisfies that for each $\mathfrak{q}$

$$
\phi_{\mathfrak{q}}(D(x))^{p-1} \in \bigoplus_{i=0}^{p-2} R_{\mathfrak{q}^{\prime}}^{\prime} \phi_{\mathfrak{q}}(x)^{i},
$$

then $D$ is preferable.

Proof. Let $\mathfrak{m}$ be a maximal ideal of $R$, and let $\left\{\phi_{\mathfrak{m}}(y)\right\} \quad(y \in R)$ be a $p$-basis of $R_{\mathfrak{m}}$ over $R_{\mathfrak{m}^{\prime}}^{\prime}$. By the same argument as in the proof of Lemma 1.1, we see that $\phi_{\mathfrak{q}}(D(y))^{p-1} \in \bigoplus_{i=0}^{p-2} R_{\mathfrak{q}^{\prime}}^{\prime} \phi_{\mathfrak{q}}(y)^{i}$ for each $\mathfrak{q}$ contained in $\mathfrak{m}$. Writing $\phi_{\mathfrak{m}}(D(y))^{p-1}$ as $\sum_{i=0}^{p-1}\left(c_{i} / s_{i}\right) \phi_{\mathfrak{m}}(y)^{i}\left(c_{i} \in R^{\prime}, s_{i} \in R^{\prime}-\mathfrak{m}^{\prime}\right)$, there exists an element $t$ of $R^{\prime}-\mathfrak{q}^{\prime}$ such that $c_{p-1} t=0$. This means that $c_{p-1} \in \bigcap_{\text {all } \mathfrak{q} \subset \mathfrak{m}} \mathfrak{q}^{\prime}$. Since $R$ is reduced, the localization $R_{\mathfrak{m}^{\prime}}^{\prime}$ of $R^{\prime}$ is also, i.e., the nilradical $\left(\bigcap_{\text {all } \mathfrak{q} \subseteq \mathfrak{m}} \mathfrak{q}^{\prime}\right) R_{\mathfrak{m}^{\prime}}^{\prime}$ is equal to $(0)$. It follows that $c_{p-1} / s_{p-1}=0$. Thus $D$ is preferable.

\section{2. $p$-BASES WHICH CONSIST OF ONE ELEMENT}

Lemma 2.1. Let $D$ be a derivation of $R$. Then, for any $a \in R$ we have

$$
(a D)^{p-1}(a)=-a D^{p-1}\left(a^{p-1}\right) .
$$

Proof. To prove this assertion, we make use of the proof of the Hochschild formula (see Theorem 25.5 of [4]). By induction, for $k \geq 1$ we get

$$
(a D)^{k}=a^{k} D^{k}+\sum_{i=2}^{k-1} b_{k, i} D^{i}+(a D)^{k-1}(a) D,
$$

where $b_{k, i}=f_{k, i}\left(a, D(a), D^{2}(a), \ldots, D^{k-i}(a)\right)(2 \leq i \leq p-1)$, more precisely the $f_{k, i}$ are polynomials with coefficients in $\mathbb{Z} /(p)$ not depending on $R$, on $a$ or on $D$. Then according to the proof of Theorem 25.5 of [4], the polynomial $f_{p, i}$ is equal to 0 for any $i$. On the other hand, the following expansion is obtained:

$$
\begin{aligned}
(a D)^{p}= & a^{p} D^{p}+a\left\{D\left(a^{p-1}\right)+b_{p-1, p-2}\right\} D^{p-1}+\sum_{i=3}^{p-2} a\left\{D\left(b_{p-1, i}\right)+b_{p-1, i-1}\right\} D^{i} \\
& +a\left\{D\left(b_{p-1,2}\right)+(a D)^{p-2}(a)\right\} D^{2}+(a D)^{p-1}(a) D .
\end{aligned}
$$

Hence, we get the following recurrence formula:

$$
\left\{\begin{aligned}
D\left(a^{p-1}\right)+b_{p-1, p-2} & =0 \\
D\left(b_{p-1, i}\right)+b_{p-1, i-1} & =0 \\
D\left(b_{p-1,2}\right)+(a D)^{p-2}(a) & =0
\end{aligned}\right.
$$

It follows that

$$
\begin{aligned}
(a D)^{p-2}(a) & =-D\left(b_{p-1,2}\right) \\
& =-D\left(-D\left(b_{p-1,3}\right)\right) \\
& \ldots \ldots \\
& =(-1)^{p-3} D^{p-3}\left(-D\left(a^{p-1}\right)\right) \\
& =(-1)^{p-2} D^{p-2}\left(a^{p-1}\right) .
\end{aligned}
$$

Consequently, we have $(a D)^{p-1}(a)=-a D^{p-1}\left(a^{p-1}\right)$. 
Theorem 2.2. Suppose $R$ is finitely presented as an $R^{\prime}$-module. Then the following conditions are equivalent:

(1) $R$ has a p-basis over $R^{\prime}$ which consists of one element.

(2) $R$ has locally $p$-bases over $R^{\prime}$ and $D_{e r} r_{R^{\prime}}(R)$ has a basis $D$ such that $D^{p}=0$.

(3) $R$ has locally p-bases over $R^{\prime}$ and $\operatorname{Der}_{R^{\prime}}(R)$ has a basis which consists of one preferable derivation.

Proof. $(1) \Rightarrow(2)$. This assertion is obvious.

$(2) \Rightarrow(3)$. Let $\mathfrak{m}$ be a maximal ideal of $R$. Since $R$ is a finitely presented $R^{\prime}$ module, the module $\operatorname{Der}_{R_{\mathfrak{w}^{\prime}}}\left(R_{\mathfrak{m}}\right)$ is canonically isomorphic to $\operatorname{Der}_{R^{\prime}}(R) \otimes_{R} R_{\mathfrak{m}}$. This implies that $\operatorname{Der}_{R_{\mathfrak{m}^{\prime}}^{\prime}}\left(R_{\mathfrak{m}}\right)$ is a free $R_{\mathfrak{m}}$-module with rank 1 . So any $p$-basis of $R_{\mathfrak{m}}$ over $R_{\mathfrak{m}^{\prime}}^{\prime}$ consists of one element. Let $\phi(x)(x \in R)$ be a $p$-basis of $R_{\mathfrak{m}}$ over $R_{\mathfrak{m}^{\prime}}^{\prime}$, where $\phi$ expresses the canonical map $R \longrightarrow R_{\mathfrak{m}}$. For the canonical map $\tau_{\mathfrak{m}}$, note that $\tau_{\mathfrak{m}}\left(D^{p}\right)=\tau_{\mathfrak{m}}(D)^{p}$. Since $D_{\phi(x)}$ forms a basis for $\operatorname{Der}_{R_{\mathfrak{m}^{\prime}}^{\prime}}\left(R_{\mathfrak{m}}\right)$, we have

$$
\tau_{\mathfrak{m}}(D)=\phi(D(x)) D_{\phi(x)} \quad \text { and } \quad \tau_{\mathfrak{m}}\left(D^{p}\right)=\phi\left(D^{p}(x)\right) D_{\phi(x)} .
$$

By virtue of Lemma 2.1, we have

$$
\phi\left(D^{p}(x)\right)=\left\{\phi(D(x)) D_{\phi(x)}\right\}^{p-1}(\phi(D(x)))=-\phi(D(x)) D_{\phi(x)}^{p-1}\left(\phi(D(x))^{p-1}\right) .
$$

Hence, the following equation is obtained:

$$
\tau_{\mathfrak{m}}\left(D^{p}\right)=-\phi(D(x)) D_{\phi(x)}^{p-1}\left(\phi(D(x))^{p-1}\right) D_{\phi(x)} .
$$

Now, $\phi(D(x))$ is a unit in $R_{\mathfrak{m}}$, because $\tau_{\mathfrak{m}}(D)$ forms a basis for $D e r_{R_{\mathfrak{m}^{\prime}}^{\prime}}\left(R_{\mathfrak{m}}\right)$. From this $D^{p}=0$ implies $D_{\phi(x)}^{p-1}\left(\phi(D(x))^{p-1}\right)=0$. Thus $D$ is preferable.

$(3) \Rightarrow(1)$. Let $\{\mathfrak{p}\}$ be the set of all prime ideals of $R$, and for each $\mathfrak{p}$ let $\{\phi(x)\}$ $(x \in R)$ be a $p$-basis of $R_{\mathfrak{p}}$ over $R_{\mathfrak{p}^{\prime}}^{\prime}$, where $\phi$ is the canonical map $R \longrightarrow R_{\mathfrak{p}}$. Let $D$ be a preferable derivation which is a basis of $\operatorname{Der}_{R^{\prime}}(R)$. Since $R$ is a finitely presented $R^{\prime}$-module, $\tau_{\mathfrak{p}}(D)$ forms a basis for $\operatorname{Der}_{R_{\mathfrak{p}^{\prime}}}\left(R_{\mathfrak{p}}\right)$ as in the proof of $(2) \Rightarrow$ $(3)$, so $D(x) \notin \mathfrak{p}$. We claim that $\operatorname{Ker} D=R^{\prime}$. Indeed, $R$ is a Galois extension of $R^{\prime}$, and the claim follows from Theorem 9 (2) of [6]. Put $f=D(x)^{p}$ and $D_{(f)}=\left\{D(x)^{p-1} / f\right\} \tau_{f}(D)$. Then $D_{(f)}$ is an element of $D e r_{R_{f}^{\prime}}\left(R_{f}\right)$ such that $\operatorname{Ker} D_{(f)}=R_{f}^{\prime}$ and $D_{(f)}\left(\phi_{f}(x)\right)=1$. Moreover, $D_{(f)}^{p}=0$, because $\tau_{f, \mathfrak{p}}\left(D_{(f)}^{p}\right)=$ $\left(\tau_{f, \mathfrak{p}}\left(D_{(f)}\right)\right)^{p}=\left(D_{\phi(x)}\right)^{p}=0$ for any prime ideal $\mathfrak{p}$ which does not contain $f$, where $\tau_{f, \mathfrak{p}}$ is the canonical map $\operatorname{Der}_{R_{f}^{\prime}}\left(R_{f}\right) \longrightarrow \operatorname{Der}_{R_{\mathfrak{p}^{\prime}}^{\prime}}\left(R_{\mathfrak{p}}\right)$. By Theorem 27.3 (i) of [4], $\left\{\phi_{f}(x)\right\}$ is a $p$-basis of $R_{f}$ over $R_{f}^{\prime}$.

Now, since Spec $R^{p}$ is quasi-compact, we can take a finite subset $\left\{f_{1}, \ldots, f_{m}\right\}$ of $\{f\}_{\mathfrak{p} \in \operatorname{Spec} R}$ and a finite subset $\left\{g_{1}, \ldots, g_{m}\right\}$ of $R^{p}$ such that $\sum_{j=1}^{m} f_{j} g_{j}=1$. Denote by $x_{j}$ the element $x$ associated with each $f_{j}$. Since $D$ is preferable, by Lemma 1.1 we have $\phi_{f_{j}}\left(D\left(x_{j}\right)\right)^{p-1} \in \bigoplus_{i=0}^{p-2} R_{f_{j}}^{\prime} \phi_{f_{j}}\left(x_{j}\right)^{i}$ for each $j$. Hence, we can write $\phi_{f_{j}}\left(D\left(x_{j}\right)\right)^{p-1}$ as $\left\{\sum_{i=0}^{p-2}(i+1) c_{i j} x_{j}^{i}\right\} / f_{j}^{n_{j}}$, where $c_{i j}(0 \leq i \leq p-1,1 \leq j \leq m)$ are elements of $R^{\prime}$ and $n_{j}(1 \leq j \leq m)$ are non-negative integers. There exists a positive integer $e$ such that $p^{e} \geq n_{j}+1$ and $f_{j}^{p^{e}-n_{j}-1}\left\{f_{j}^{n_{j}} D\left(x_{j}\right)^{p-1}-\sum_{i=0}^{p-2}(i+1) c_{i j} x_{j}^{i}\right\}=0$ 
for all $j$. Here, put $z=\sum_{j=1}^{m} g_{j}^{p^{e}}\left(\sum_{i=0}^{p-2} f_{j}^{p^{e}-n_{j}-1} c_{i j} x_{j}^{i+1}\right)$. Then we have

$$
\begin{aligned}
D(z) & =\sum_{j=1}^{m} g_{j}^{p^{e}}\left\{\sum_{i=0}^{p-2}(i+1) f_{j}^{p^{e}-n_{j}-1} c_{i j} x_{j}^{i}\right\} D\left(x_{j}\right) \\
& =\sum_{j=1}^{m} g_{j}^{p^{e}}\left(f_{j}^{p^{e}-1} D\left(x_{j}\right)^{p-1}\right) D\left(x_{j}\right) \\
& =\sum_{j=1}^{m} f_{j}^{p^{e}} g_{j}^{p^{e}}=1 .
\end{aligned}
$$

Now, we shall show that $\{z\}$ is a $p$-basis of $R$ over $R^{\prime}$. According to Theorem 27.3 (i) of [4], nothing remains but to show $D^{p}=0$. Since $D$ forms a basis for $\operatorname{Der}_{R^{\prime}}(R)$, the derivation $D^{p}$ is equal to $a D(a \in R)$. Clearly, $a=a D(z)=D^{p}(z)=0$. Thus $D^{p}=0$. Therefore $R$ has the $p$-basis $\{z\}$ over $R^{\prime}$.

Corollary 2.3. Suppose that $R$ and $R^{\prime}$ are regular noetherian rings, and suppose that $R$ is finitely generated as an $R^{\prime}$-module. Then the following conditions are equivalent:

(1) $R$ has a p-basis over $R^{\prime}$ which consists of one element.

(2) $\operatorname{Der}_{R^{\prime}}(R)$ has a basis $D$ such that $D^{p}=0$.

(3) $\operatorname{Der}_{R^{\prime}}(R)$ has a basis which consists of one preferable derivation.

Proof. By the Theorem of [1] (cf. [3], Theorem 15.7), $R$ has locally $p$-bases over $R^{\prime}$. Therefore this is an immediate consequence of Theorem 2.2 .

\section{3. $p$-BASES WHICH CONSIST OF $l$ ELEMENTS}

Lemma 3.1. Suppose that $R$ is a Galois extension of $R^{\prime}$. Let $D$ be a derivation of $R$ over $R^{\prime}$, and suppose that $D^{p}=0$ and the $R$-module $R D$ is a direct summand of $\operatorname{Der}_{R^{\prime}}(R)$. Then the following holds:

(1) $R$ is a Galois extension of $\operatorname{Ker} D$,

(2) $\operatorname{Ker} D$ is a Galois extension of $R^{\prime}$,

(3) $R D=\operatorname{Der}_{K e r} D(R)$.

Proof. For any $a, b \in R$, we have

$$
[a D, b D]=\{a D(b)-b D(a)\} D,
$$

and by the Hochschild formula

$$
(a D)^{p}=a^{p} D^{p}+(a D)^{p-1}(a) D=(a D)^{p-1}(a) D .
$$

Thus $[a D, b D]$ and $(a D)^{p}$ are contained in $R D$. It follows that $R D$ is a $p$-Lie subalgebra of $\operatorname{Der}_{R^{\prime}}(R)$. Theorem 12 of [6] says that $R$ is a Galois extension of $\operatorname{Ker} D$ and $R D=\operatorname{Der}_{\operatorname{Ker} D}(R)$. Therefore $\operatorname{Ker} D$ is a Galois extension of $R^{\prime}$ by Theorem 11 of [6].

Theorem 3.2. Let $l$ be an integer greater than 1 . Suppose $R$ is finitely presented as an $R^{\prime}$-module. Then the following conditions are equivalent:

(1) $R$ has a p-basis over $R^{\prime}$ which consists of $l$ elements.

(2) $R$ has locally $p$-bases over $R^{\prime}$ and $\operatorname{Der}_{R^{\prime}}(R)$ has a basis $\left\{D_{1}, \ldots, D_{l}\right\}$ such that $D_{i}^{p}=0$ and $\left[D_{i}, D_{j}\right]=0$ for any $i, j=1,2, \ldots, l$. 
Proof. (1) $\Rightarrow(2)$. This immediately follows from fact (3) in $\S 1$.

$(2) \Rightarrow(1)$. Let $R_{1}$ be the kernel of the derivation $D_{1}$ which is an $R^{\prime}$-algebra. Then, by Lemma $3.1 R$ is a Galois extension of $R_{1}$ and $R D_{1}=\operatorname{Der}_{R_{1}}(R)$. Hence, there exists a $p$-basis $\left\{x_{1}\right\}$ of $R$ over $R_{1}$ by Theorem 2.2 .

Now, in order to find the other elements which constitute a $p$-basis of $R$ over $R^{\prime}$, we need to show that $\left\{\left.D_{i}\right|_{R_{1}}\right\}_{i=2, \ldots, l}$ forms a basis for $\operatorname{Der}_{R^{\prime}}\left(R_{1}\right)$. First of all, we claim that $\left.D_{i}\right|_{R_{1}} \in \operatorname{Der}_{R^{\prime}}\left(R_{1}\right)$ and $\left.D_{i}\right|_{R_{1}} \neq 0$ for any $i \geq 2$. The first assertion follows from $\left[D_{1}, D_{i}\right]=0$. To show the second assertion, assume $R_{1} \subseteq \operatorname{Ker} D_{i}$. Then $D_{i} \in \operatorname{Der}_{R_{1}}(R)=R D_{1}$. This contradicts the fact that $\left\{D_{1}, \ldots, D_{l}\right\}$ is a basis of $\operatorname{Der}_{R^{\prime}}(R)$. Thus $\left.D_{i}\right|_{R_{1}} \neq 0$. Let $\mathfrak{m}$ be a maximal ideal of $R$ and let $\mathfrak{n}$ be the maximal ideal $\mathfrak{m} \cap R_{1}$ of $R_{1}$. Since $R_{1}$ is a Galois extension of $R^{\prime}$ by Lemma 3.1 , there is a subset $\left\{y_{2}, \ldots, y_{l}\right\}$ of $R_{1 \mathfrak{n}}$ which is a $p$-basis of $R_{1 \mathfrak{n}}$ over $R_{\mathfrak{m}^{\prime}}^{\prime}$. Obviously, $\left\{\phi_{\mathfrak{m}}\left(x_{1}\right), y_{2}, \ldots, y_{l}\right\}$ is a $p$-basis of $R_{\mathfrak{m}}$ over $R_{\mathfrak{m}^{\prime}}^{\prime}$. Let $D_{\phi_{\mathfrak{m}}\left(x_{1}\right)}, D_{y_{2}}, \ldots, D_{y_{l}}$ be the derivations of $R_{\mathfrak{m}}$ over $R_{\mathfrak{m}^{\prime}}^{\prime}$ associated with this $p$-basis (see fact (3) in $\S 1$ ). Denote by $D_{j}^{\prime}$ the derivation $\left.D_{y_{j}}\right|_{R_{1 \mathfrak{n}}}$ of $R_{1 \mathfrak{n}}$ over $R_{\mathfrak{m}^{\prime}}^{\prime}$. Then $\tau_{\mathfrak{n}}\left(\left.D_{i}\right|_{R_{1}}\right)$ is written as $\sum_{j=2}^{l} a_{i j} D_{j}^{\prime}$ for each $i \geq 2$ where $a_{i j} \in R_{1 \mathfrak{n}}$, because $\left\{D_{j}^{\prime}\right\}_{j=2, \ldots, l}$ forms a basis for $\operatorname{Der}_{R_{\mathfrak{m}^{\prime}}^{\prime}}\left(R_{1 \mathfrak{n}}\right)$. Since $R$ is finitely presented as an $R^{\prime}$-module, the module $\operatorname{Der}_{R_{\mathfrak{m}^{\prime}}^{\prime}}\left(R_{\mathfrak{m}}\right)$ is isomorphic to $\operatorname{Der}_{R^{\prime}}(R) \otimes_{R} R_{\mathfrak{m}}$. Hence, $\left\{\tau_{\mathfrak{m}}\left(D_{1}\right), \ldots, \tau_{\mathfrak{m}}\left(D_{l}\right)\right\}$ forms a basis for $\operatorname{Der}_{R_{\mathfrak{m}^{\prime}}^{\prime}}\left(R_{\mathfrak{m}}\right)$, so the derivation $D_{y_{j}}$ is expressed as $\sum_{i=1}^{l} b_{j i} \tau_{\mathfrak{m}}\left(D_{i}\right)$ for each $j \geq 2$ where $b_{j i} \in R_{\mathfrak{m}}$. For each $j \geq 2$ we have

$$
D_{j}^{\prime}=\left.\sum_{i=1}^{l} b_{j i} \tau_{\mathfrak{m}}\left(D_{i}\right)\right|_{R_{1 \mathfrak{n}}}=\sum_{i=2}^{l} b_{j i} \tau_{\mathfrak{n}}\left(\left.D_{i}\right|_{R_{1}}\right) .
$$

These show that the matrix $\left[b_{j i}\right]_{2 \leq i, j \leq l}$ is equal to the inverse matrix of $\left[a_{i j}\right]_{2 \leq i, j \leq l}$, i.e., $b_{j i} \in R_{1 \mathfrak{n}}$. Thus, for any maximal ideal $\mathfrak{n}$ of $R_{1},\left\{\tau_{\mathfrak{n}}\left(\left.D_{i}\right|_{R_{1}}\right)\right\}_{i=2, \ldots, l}$ is a basis of $\operatorname{Der}_{R^{\prime}}\left(R_{1 \mathfrak{n}}\right)$. This implies that $\left\{\left.D_{i}\right|_{R_{1}}\right\}_{i=2, \ldots, l}$ forms a basis for $\operatorname{Der}_{R^{\prime}}\left(R_{1}\right)$.

Set $R_{h}=\operatorname{Ker} D_{1} \cap \cdots \cap \operatorname{Ker} D_{h}$ for $h=2, \ldots, l$. Repeating the previous argument in the situation that $R_{h-1} \supseteq R_{h} \supseteq R^{\prime}$, we can show that there exists a $p$-basis $\left\{x_{h}\right\}$ of $R_{h-1}$ over $R_{h}$ inductively. Then Theorem 9 (2) of [6] says that $R_{l}=R^{\prime}$. In conclusion, $\left\{x_{1}, \ldots, x_{l}\right\}$ is a $p$-basis of $R$ over $R^{\prime}$.

Corollary 3.3. Let $l$ be an integer greater than 1 . Suppose that $R$ and $R^{\prime}$ are regular noetherian rings, and suppose that $R$ is finitely generated as an $R^{\prime}$-module. Then the following are equivalent:

(1) $R$ has a p-basis over $R^{\prime}$ which consists of $l$ elements.

(2) $\operatorname{Der}_{R^{\prime}}(R)$ has a basis $\left\{D_{1}, \ldots, D_{l}\right\}$ such that $D_{i}^{p}=0$ and $\left[D_{i}, D_{j}\right]=0$ for any $i, j=1,2, \ldots, l$.

Proof. By virtue of the Theorem of [1], $R$ has locally $p$-bases over $R^{\prime}$. Clearly, the assertion holds by Theorem 3.2 .

\section{4. $p$-BASES OF POLYNOMIAL RINGS}

In this section, when $R$ is an integral domain, $Q(R)$ denotes the field of fractions of $R$. The next theorem is an analogy of the result of [2].

Theorem 4.1. Let $k$ be a perfect field of characteristic $p>0$. Let $A$ be a polynomial ring $k\left[X_{1}, \ldots, X_{n}\right]$, and let $B$ be a regular noetherian subring of $A$ containing $A^{p}$ such that $\left[Q(B): Q\left(A^{p}\right)\right]=p$. Suppose that $\operatorname{Der}_{A^{p}}(B)$ is a free B-module. If $F$ 
is a polynomial of minimal degree $\left(\right.$ in $\left.X_{1}, \ldots, X_{n}\right)$ in $B-A^{p}$ which has no terms of elements in $A^{p}$, then $\{F\}$ is a p-basis of $B$ over $A^{p}$.

Proof. Since $A$ is finitely generated as an $A^{p}$-module and $B$ is noetherian, $A$ is finitely presented as a $B$-module. By the Theorem of [1], $A$ is a Galois extension of $B$. Clearly $A$ is a Galois extension of $A^{p}$, and $B$ is also by Theorem 11 (1) of [6].

Set $H=\left\{D \in \operatorname{Der}_{A^{p}}(A) \mid D(B) \subseteq B\right\}$. Then, by Theorem 11 (2) of [6], there is a $B$-module homomorphism $\Phi: \operatorname{Der}_{A^{p}}(B) \longrightarrow H$ which, followed by the restriction map $H \longrightarrow D e r_{A^{p}}(B)$ given by $\left.D \longrightarrow D\right|_{B}$, is the identity map on $\operatorname{Der}_{A^{p}}(B)$. We write $\operatorname{Der}_{A^{p}}(B)$ for the image of $\operatorname{Der}_{A^{p}}(B)$ in $H$. Theorem 11 (3) of [6] says that

$$
\operatorname{Der}_{A^{p}}(A)=\operatorname{Der}_{B}(A) \oplus A \widetilde{\operatorname{Der}_{A^{p}}(B)} .
$$

We see that $\operatorname{rank}_{B} \operatorname{Der}_{A^{p}}(B)=1$, because $\left[Q(B): Q\left(A^{p}\right)\right]=p$. Let $D$ be a basis for $\operatorname{Der}_{A^{p}}(B)$, and put $\widetilde{D}=\Phi(D)$. Obviously $\left.\widetilde{D}\right|_{B}=D$, so $\widetilde{D}$ generates $\widetilde{\operatorname{Der}_{A^{p}}(B)}$. From $(*)$, there are a derivation $D_{i} \in \operatorname{Der}_{B}(A)$ and an element $a_{i} \in A$ such that

$$
\frac{\partial}{\partial X_{i}}=D_{i}+a_{i} \widetilde{D} \quad \text { for } i=1, \ldots, n .
$$

Hence, for each $i$ we have

$$
\frac{\partial F}{\partial X_{i}}=a_{i} \widetilde{D}(F)
$$

Now, $F \notin A^{p}$ implies $a_{j} \neq 0$ for some $j$. It follows that

$$
\operatorname{deg} \widetilde{D}(F) \leq \operatorname{deg} \frac{\partial F}{\partial X_{j}}<\operatorname{deg} F \text {. }
$$

On the other hand, since $F \in B-A^{p}$ and $\operatorname{Ker} D=A^{p}$ (see [6], Theorem 9 (2)), we obtain

$$
\widetilde{D}(F)=\left.\widetilde{D}\right|_{B}(F)=D(F) \in B-\{0\} .
$$

Since the degree of $F$ is minimal in $B-A^{p}$, the above $(\dagger)$ and ( $\ddagger$ ) yield that

$$
D(F) \in A^{p}-\{0\} .
$$

Let $t(t \in B)$ be a $p$-basis of $Q(B)$ over $Q\left(A^{p}\right)$, and let $D_{t}$ be a derivation of $Q(B)$ over $Q\left(A^{p}\right)$ such that $D_{t}(t)=1$. Then, since $D_{t}$ is a basis of $\operatorname{Der}_{Q\left(A^{p}\right)}(Q(B))$, the derivation $D$ is equal to $D(t) D_{t}$, where $D$ is regarded as the derivation of $Q(B)$ over $Q\left(A^{p}\right)$ by the canonical inclusion map $\operatorname{Der}_{A^{p}}(B) \longrightarrow \operatorname{Der}_{Q\left(A^{p}\right)}(Q(B))$. So we have

$$
D(t)^{p-1}=\frac{1}{D(F)} D(t)^{p} D_{t}(F) \in \bigoplus_{i=0}^{p-2} Q\left(A^{p}\right) t^{i}
$$

Hence, $D$ is preferable by Lemma 1.2. According to the proof of Theorem 2.2, there exists a $p$-basis $\left\{F^{\prime}\right\}$ of $B$ over $A^{p}$ such that $D\left(F^{\prime}\right)=1$. We may assume that $F^{\prime}$ has no terms of elements in $A^{p}$. Writing $F$ as $\sum_{i=0}^{p-1} a_{i}^{p}{F^{\prime}}^{i}\left(a_{i} \in A\right),(\star)$ implies that $a_{2}=a_{3}=\cdots=a_{p-1}=0$. Considering the assumptions for the degree and the terms of $F$, we have $a_{0}=0$ and $a_{1} \in k-\{0\}$. Consequently, $\{F\}$ is a $p$-basis of $B$ over $A^{p}$.

Remark. The following assertions immediately follow from the proof of Theorem 4.1.

(1) $F$ is unique up to multiplication by elements of $k-\{0\}$. 
(2) Any $p$-basis of $B$ over $A^{p}$ can be uniquely expressed as $c F+a^{p}(c \in k-$ $\{0\}, a \in A)$.

Corollary 4.2 (Kimura-Niitsuma). Let $k$ and $A$ be as in Theorem 4.1. Let $B$ be a polynomial ring $k\left[Y_{1}, \ldots, Y_{n}\right]$ which is a subring of $A$ containing $A^{p}$ such that $\left[Q(B): Q\left(A^{p}\right)\right]=p($ resp. $[Q(A): Q(B)]=p)$. Then $B$ has a $p$-basis over $A^{p}$ (resp. $A$ has a p-basis over $B)$.

Proof. Suppose $\left[Q(B): Q\left(A^{p}\right)\right]=p$. Recall that $B$ is a Galois extension of $A^{p}$ (see the proof of Theorem 4.1). By Theorem 9 of [6] $\operatorname{Der}_{A^{p}}(B)$ is afinitely generated and projective as a $B$-module. By virtue of Quillen's result of [5], $\operatorname{Der}_{A^{p}}(B)$ is free. Therefore the assertion holds by Theorem 4.1.

Next, suppose $[Q(A): Q(B)]=p$. Then by a similar argument we can show that there is a $p$-basis $\left\{F^{p}\right\}(F \in A)$ of $A^{p}$ over $B^{p}$. Obviously, $\{F\}$ is a $p$-basis of $A$ over $B$.

Remark. In 1990, the above result was first announced by T. Kimura and H. Niitsuma.

\section{REFERENCES}

[1] T. Kimura and H. Niitsuma, On Kunz's conjecture, J. Math. Soc. Japan 34 (1982), 371-378. MR 83h:13030

[2] _ A note on p-basis of polynomial ring in two variable, SUT J. Math. 25 (1989), 33-38. MR 91a:13002

[3] E. Kunz, Kähler Differentials, Vieweg Advanced Lectures in Math., 1986. MR 88e:14025

[4] H. Matsumura, Commutative Ring Theory, Cambridge University Press, Cambridge, 1986. MR 88h:13001

[5] D. Quillen, Projective modules over polynomial rings, Invent. Math. 36 (1976), 167-171. MR 55:337

[6] S. Yuan, Inseparable Galois theory of exponent one, Trans. Amer. Math. Soc. 149 (1970), 163-170. MR 41:1717

Tokyo Metropolitan College of Aeronautical Engineering 8-52-1, Minami-Senju, ARAKAWA-KU, TOKYO 116-0003, JAPAN

E-mail address: tono@kouku-k.ac.jp 